\title{
Evaluation of waveform data processing in Wave-Particle Interaction Analyzer
}

\author{
Mitsuru Hikishima ${ }^{1,2^{*}}$, Yuto Katoh ${ }^{1}$ and Hirotsugu Kojima ${ }^{3}$
}

\begin{abstract}
The Wave-Particle Interaction Analyzer (WPIA) is a software function installed on the Exploration of energization and Radiation in Geospace (ERG) satellite. The WPIA directly measures the quantity of energy transfer between whistler-mode chorus waves and resonant energetic electrons by using plasma wave vectors and velocity vectors of plasma particles. The phase differences of the WPIA require accurate phase angles of waves and electrons in order to statistically evaluate the significance of the quantity of energy transfer. We propose a technical method for efficient waveform processing in order to conduct the WPIA measurement precisely. In the WPIA measurement, the various waves detected by the onboard instrument appear as noise in the calculation of the quantity of energy transfer for whistler-mode chorus waves. The characteristic frequency variation of the chorus waves makes waveform processing difficult. A chorus waveform is used for the WPIA processing through passband filtering by selecting appropriate data processing length and frequency resolution. We implement overlapping processing of wave data in order to reduce the induced error of the wave phase. The results of waveform processing indicate that the phase errors are successfully reduced and statistical fluctuations are suppressed. The proposed waveform processing method is a necessary and applicative processing for the calculations of the WPIA in the ERG mission.
\end{abstract}

Keywords: Whistler-mode chorus; Wave-particle interaction; Waveform processing

\section{Background}

The Wave-Particle Interaction Analyzer (WPIA) is an instrument package installed in the Exploration of energization and Radiation in Geospace (ERG) satellite (Miyoshi et al. 2013). The WPIA, which directly measures the energy transfer in the process of wave-particle resonant interactions between plasma waves and charged particles in the space plasma environment, is equipped as a software function. Whistler-mode chorus waves are commonly observed outside the plasmasphere, and the waves can cause resonant interactions with energetic electrons in the kinetic energy range from a few $\mathrm{keV}$ to a few $\mathrm{MeV}$. The whistler-mode chorus waves are also considered to be a candidate for inducing relativistic electrons of $\mathrm{MeV}$ energy during a magnetic storm in the inner magnetosphere (e.g., Summers et al. 1998; Meredith et al. 2002).

\footnotetext{
*Correspondence: hikishima@stpp.gp.tohoku.ac.jp

1 Department of Geophysics, Graduate School of Science, Tohoku University,

Miyagi 980-8578, Japan

22 Solar-Terrestrial Environment Laboratory, Nagoya University, Nagoya, Aichi 464-8601, Japan

Full list of author information is available at the end of the article
}

In the ERG mission, the primary goal is to clarify the acceleration process of plasma particles by wave-particle interactions in the inner magnetosphere. The WPIA is the first attempt to directly measure the existence of energy transfer between whistler-mode chorus waves and resonant energetic electrons in space.

The WPIA uses an instantaneous wave field vector and the velocity vector of each electron, which are measured using wave and particle instruments with microsecond resolution onboard the ERG satellite. The energy transfer $W$, which is represented by the inner product of $\boldsymbol{E}$ and $\boldsymbol{v}$, where $\boldsymbol{E}$ and $\boldsymbol{v}$ are the wave electric field vector and the velocity vector of an electron, respectively (Fukuhara et al. 2009). The resonant interactions between whistler-mode chorus waves and electrons are investigated in the WPIA measurement. Whistler-mode chorus waves are generated around the equator outside the plasmapause during geomagnetic disturbances (Tsurutani and Smith 1974). Whistler-mode chorus waves appear in the frequency range below the electron gyrofrequency $f_{\text {ce }}$ and have distinct upper and lower frequency bands, which are identified by a gap of $0.5 f_{\text {ce }}$ (Burtis

\section{它 Springer}

(c) 2014 Hikishima et al: licensee Springer. This is an Open Access article distributed under the terms of the Creative Commons Attribution License (http://creativecommons.org/licenses/by/2.0), which permits unrestricted use, distribution, and reproduction in any medium, provided the original work is properly credited. 
and Helliwell 1969; Tsurutani and Smith 1974). Magnetosonic waves (MSW) and electron cyclotron harmonic $(\mathrm{ECH})$ waves are often observed with the chorus waves in a region close to the magnetic equator. The MSW are equatorial electromagnetic waves that have frequencies below the lower-hybrid frequency $f_{\mathrm{LHR}}$ (e.g., a few hundred hertz at $L \sim 4$ ) (Santolík et al. 2002). The intensity approximately $200 \mu \mathrm{V} / \mathrm{m}$ is comparable to the chorus wave amplitude (Gurnett 1976). The intense ECH waves are electrostatic waves and also appear with the excitation of chorus waves near the magnetic equator. The frequencies have harmonic components in $(n+1 / 2) f_{\text {ce }}(n=$ $1,2, \ldots)$ Kennel et al. (1970) with a magnitude of a few $\mathrm{mV} / \mathrm{m}$ (Meredith et al. 2009). The WPIA measures resonant interactions between whistler-mode chorus waves and resonant electrons near the equatorial region. The $\mathrm{ECH}$ and MSW are also detected with chorus waves in the region. Such intense waves affect the wave phase of chorus waves because they appear as a superposition of waves.

In the present paper, we propose a waveform processing technique, which is required in the WPIA to accurately measure the energy transfer generated by resonant waveparticle interactions. We evaluate the effectiveness of data processing using a representative model of the chorus observed near the equator. We then present an appropriate data processing method using passband filtering and an overlapping method in the WPIA. The effectiveness of the applied waveform processing is evaluated through pseudo-WPIA measurements by the electromagnetic particle simulation.

\section{Methods}

\section{The WPIA method}

The fundamental concept of the WPIA method is described in Fukuhara et al. (2009). The WPIA uses the wave field vector and the velocity vector of plasma particles, which are measured by wave and particle instruments with microsecond resolution. In order to evaluate the energy transfer, we consider the time variation of the kinetic energy of particles. The relativistic kinetic energy of a charged particle is given by $K=m_{0} c^{2}(\gamma-1)$, where $m_{0}$ and $c$ are the rest mass and the speed of light, respectively, and $\gamma$ is the Lorentz factor. The time variation of the kinetic energy is represented by the quantity $W$ as follows:

$$
W=\frac{\mathrm{d} K}{\mathrm{~d} t}=m_{0} \boldsymbol{v} \cdot \frac{\mathrm{d}(\gamma \boldsymbol{v})}{\mathrm{d} t}=q \boldsymbol{E} \cdot \boldsymbol{v},
$$

where $\boldsymbol{v}$ and $q$ are the velocity vector and the charge of the charged particle, respectively, and $\boldsymbol{E}$ is the wave's electric field vector. The WPIA calculates the inner product using the instantaneous wave electric field vector and the velocity vector of each charged particle. Figure 1 shows a schematic diagram of whistler-mode chorus waves and resonant electrons. We consider the energy transfer between waves and particles in the plane perpendicular to the ambient magnetic field line. We assume the electric and magnetic components $\left(\boldsymbol{E}_{\mathrm{w}}\right.$ and $\left.\boldsymbol{B}_{\mathrm{w}}\right)$ of transverse wave fields of the whistler-mode chorus waves and gyrating electrons with velocity $\boldsymbol{v}_{\perp}$. We define the relative phase angle $\theta$ between the wave electric field $\boldsymbol{E}_{\mathrm{w}}$ and the electron velocity $\boldsymbol{v}_{\perp}$. In the WPIA, the phase angle is important for determining the energy transfer $W$. A positive $W$ indicates the acceleration of electrons by the electric field of the wave, whereas a negative $W$ indicates the deceleration of electrons and causes wave growth. The WPIA calculates the quantity $W$ for an individual particle using the wave field at the moment the particle is detected. If there exist no resonant particles, the values of $W$ should fluctuate randomly about zero. We statistically evaluate the amount of energy transfer by summing the quantity $W$ of the detected particle over a time interval. The statistical significance of the obtained value is evaluated based on the standard deviation $\sigma_{\mathrm{w}}$ represented by the following formula (Katoh et al. 2013):

$$
\sigma_{\mathrm{w}}=\sqrt{\sum_{i=1}^{N}\left(q \boldsymbol{E}_{\mathrm{w}}\left(t_{i}\right) \cdot \boldsymbol{v}_{i}\right)^{2}-\frac{1}{N}\left(\sum_{i=1}^{N} q \boldsymbol{E}_{\mathrm{w}}\left(t_{i}\right) \cdot \boldsymbol{v}_{i}\right)^{2}},
$$

where $N$ is the total number of particles and $\boldsymbol{v}_{i}$ is the velocity of the $i$-th particle detected at time $t_{i}$.

\section{Results and discussion \\ Waveform processing for calculation in the WPIA Wave data used in the WPIA processing}

The specifications of the wave and particle instruments onboard the ERG satellite are described in Miyoshi et al. (2013). We introduce the measured wave data with respect to the waveform processing. Electric and magnetic sensors are installed on the ERG satellite in order to observe alternating plasma waves in space. Two wire probe antennas, which receive electric field components of plasma waves are attached in an orthogonal manner to the spin axis of the satellite. The wire antennas are sensitive to a wave vector of the electric field, where one electric field component along the spin axis is missing. For detection of the magnetic field components of waves, a three-axis search coil magnetometer, which is located on top of the mast, can detect magnetic components of the plasma wave field.

Figure 2 shows the process flow of the WPIA, which uses wave components of the electric and magnetic fields $\left(E_{1}, E_{2}, B_{1}, B_{2}\right.$, and $\left.B_{3}\right)$ of plasma waves picked up by sensors. The waves received by the wave sensors are transferred to the main electronic receiver, which contains amplifiers and passband filters. Signals are sampled with a specific sampling period as a digital signal. In the WPIA, 


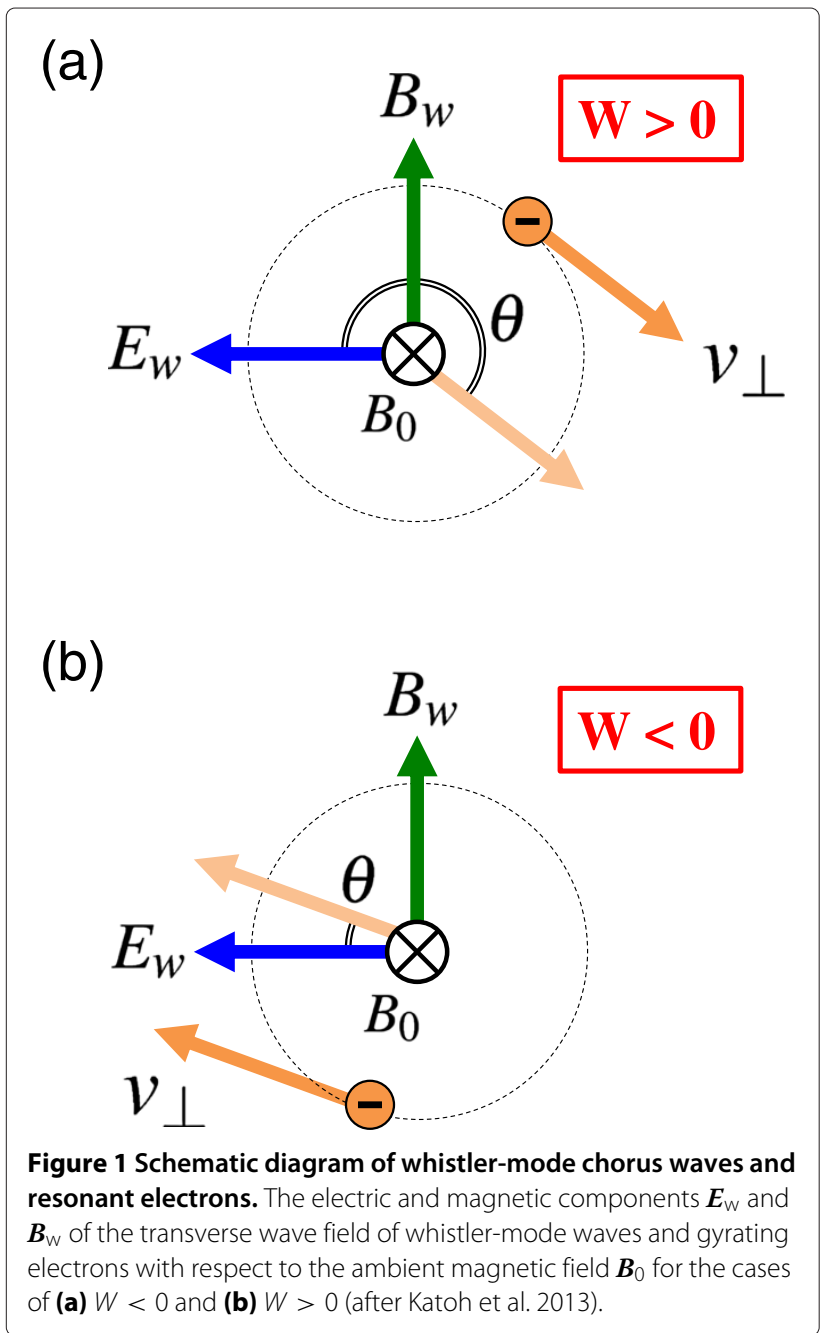

in order to obtain the amplitude and phase of the waveform detected by the sensors, the signals require calibration using the transfer functions that were applied to the signals. Since the calibration procedure is implemented in the frequency domain, waves must be transformed using the fast Fourier transform (FFT). After the calibration procedure, the original waveforms are obtained through the inverse FFT (IFFT).

The WPIA calculation uses waveforms in the frequency range of up to $20 \mathrm{kHz}$ for both the electric and magnetic wave fields. The upper frequency limit is determined by the sampling frequency of $65 \mathrm{kHz}$. Moreover, an electric waveform of up to $120 \mathrm{kHz}$ with a higher sampling frequency is prepared as a special mode in order to obtain the amplitude and phase of the waveform with better time resolution. The WPIA calculation uses data of the chorus waves. However, in the frequency range of up to $20 \mathrm{kHz}$, the MSW in hundreds of hertz and the $\mathrm{ECH}$ waves in the kilohertz frequency band are expected to be

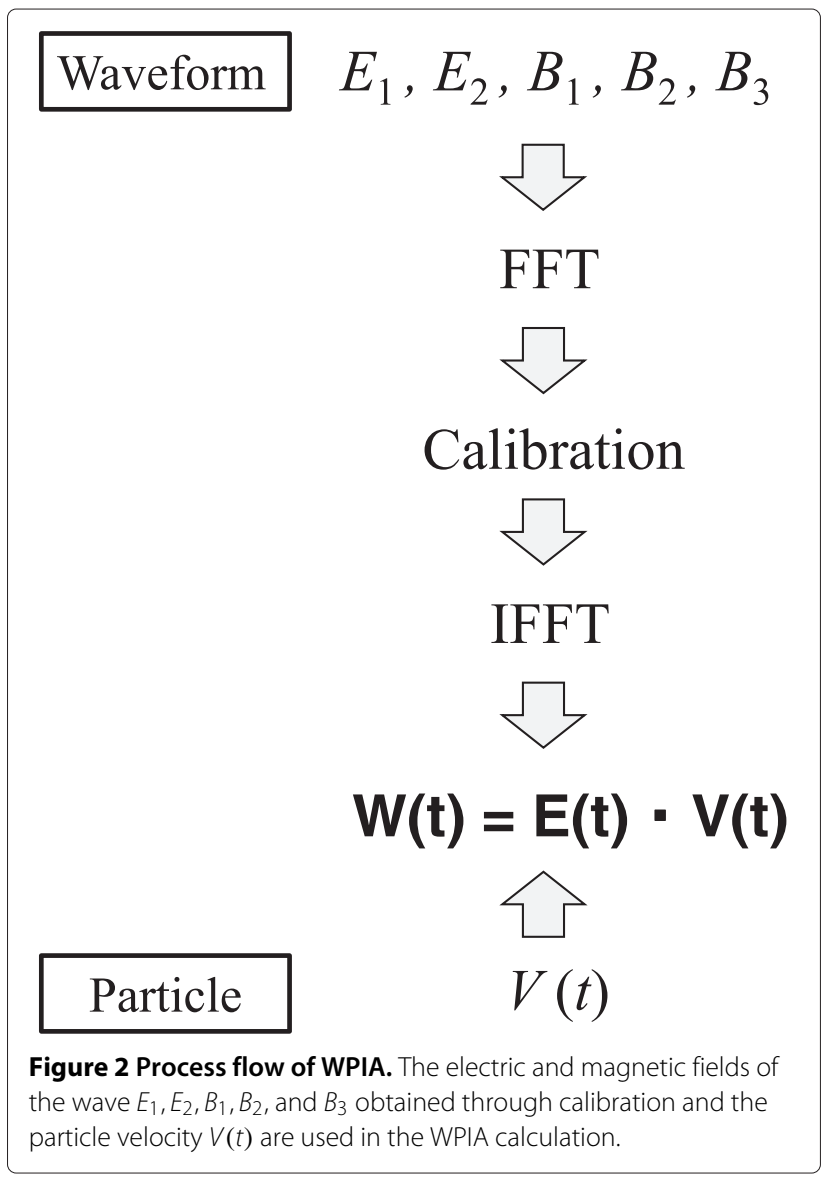

included in the waveforms observed by the ERG satellite, which passes outside the plasmapause. Such waves are observed by the sensors as superposed waves. Intense waves result in uncertainty fluctuation in the calculation of the WPIA. Therefore, the unnecessary wave components must be removed from the received waveforms through an appropriate processing.

\section{Evaluation of phase error in waveform processing}

The representative time duration of each element of chorus waves is approximately $100 \mathrm{~ms}$. The FFT processing must be implemented within this time duration. We examine the influence of the FFT processing on the waveform using a simple model chorus wave. Whistler-mode chorus waves are excited below the electron gyrofrequency near the equator (Burtis and Helliwell 1969; Tsurutani and Smith 1974). Chorus waves are observed dominantly as a rising-tone wave. We assume the simple model chorus wave near $L=4$. The waveform has sinusoidal time-varying characteristics, starting from $20 \mathrm{~ms}$. Assuming the combination of the lower- and upper-band chorus waves, the frequency increases linearly from 1 to 
Table 1 Parameters of the model chorus wave

\begin{tabular}{lc}
\hline Parameter & Value \\
\hline Sampling frequency & $65 \mathrm{kHz}$ \\
Electron gyrofrequency at the equator: $f_{\text {ce }}$ & $10 \mathrm{kHz}(\mathrm{L} \sim 4)$ \\
Chorus frequency (including upper & 1 to $9 \mathrm{kHz}\left(0.1\right.$ to $\left.0.9 f_{\mathrm{ce}}\right)$ \\
and lower bands) & $40 \mathrm{kHz} / \mathrm{s}$ \\
Frequency sweep rate: $\mathrm{d} f / \mathrm{d} t$ & $200 \mathrm{~ms}$ \\
Duration time of a chorus element & Constant \\
\hline Amplitude &
\end{tabular}

$9 \mathrm{kHz}$ with a frequency sweep rate of $40 \mathrm{kHz} / \mathrm{s}$. The duration of the model chorus is $200 \mathrm{~ms}$. The parameters of the model chorus wave are listed in Table 1.

In conducting the FFT processing, we must consider the appropriate data length of one frame used in the FFT. Since the calculation in the WPIA requires only the chorus waveform, we remove the wave mode components of the MSW and ECH waves. In order to remove the waves, we implement the passband filter applying a rectangular window with a passband of $0.1<f<1.0 f_{\text {ce. }}$ The MSW waves are observed in a frequency range of a few hundred kilohertz at $L \sim 4$ (Santolík et al. 2002), and the ECH waves appear in the frequency range of $f>f_{\text {ce }}$ (Kennel et al. 1970). In order to distinguish these waves from chorus waves in the frequency domain, a frequency resolution of better than $0.1 f_{\text {ce }}$ is required. Considering the spectral spreading of the waves, we assume a frequency resolution of a few hundred hertz. In order to satisfy the above conditions, we assume a frequency resolution of $127 \mathrm{~Hz}$, corresponding to 512 data points and a time duration of 7.8 $\mathrm{ms}$ in an FFT frame. A longer FFT length provides a more accurate frequency resolution and limits the increase in computation time due to repeated FFT processing, but results in an increased memory requirement.

Figure 3 shows a time-frequency spectrogram of the model chorus wave. The sequence of FFTs is implemented for the waveform without overlapping of the frames. The time and frequency resolutions are $7.8 \mathrm{~ms}$ and $127 \mathrm{~Hz}$, respectively. In addition, passband filtering in the frequency range of $0.1<f<1.0 f_{\text {ce }}$ is applied for each frame. The upper and lower cutoff frequencies are indicated by dashed lines. We then allow the power spectral density of chorus waves to become zero outside the frequency range. The spectral bins of rising chorus waves vary with the frequency step of $\mathrm{d} f / \mathrm{d} t \times 7.8 \mathrm{~ms}$. When using a rectangular window, the resulting frequency spectrum spreads in a wide frequency range due to characteristics of the nonperiodic waveform. In this wave model, the frequency of the chorus waves is assumed to be from 0.1 to $0.9 f_{\text {ce }}$ and subsequently increases over time. Since the broadening of the power spectral density occurs through FFT processing, the power of the chorus waves is lost by the passband filtering. The effect of the reduction is significant at frequencies close to the cutoff frequencies of the passband filter.

After processing the passband filtering in the frequency domain, we transform the spectra to a waveform in the time domain by applying the IFFT. Figure 4 shows the amplitudes and the phase angle difference between the original waveform and the waveform after passband filtering in the time interval of $t=123$ to $135 \mathrm{~ms}$, which corresponds to a chorus element of approximately $f=$ $5 \mathrm{kHz}$. The instantaneous phase angle at each sampling time is calculated from the wave amplitude, assuming a sinusoidal time-varying waveform. The dots in Figure 4a indicate the sampling points. In Figure $4 \mathrm{a}$, as a result of passband filtering, the wave amplitudes around the edges of the FFT frame ( $t=126$ and $134 \mathrm{~ms}$ ), which is shown at the top of Figure 4 , are remarkably perturbed. The phase differences are then visible as errors (Figure $4 b$ ). Since the time interval shown in Figure 4 ( $t=125$ to $135 \mathrm{~ms}$ ) corresponds to the central frequency of the model chorus wave, the removal effect of the power spectrum in the tail part is reduced. However, the phase angle errors below approximately $5^{\circ}$ exist over the entire waveform. In particular, the phase errors are maximized at the edges of a frame, whereas the error reaches up to $40^{\circ}$. In the WPIA, the accuracy of the relative phase angle is important for the calculation of the inner product of the wave vector and the particle velocity vector. Since phase angle errors generate perturbations for a quantity of calculated energy transfer, the error should be removed for calculation in the WPIA.

\section{Overlapping processing}

We consider the efficient processing of wave data for the reduction of the errors. The phase error of a waveform after IFFT processing occurs due to the implementation of passband filtering in the frequency domain. The operation of taking a rectangular window in each FFT frame results in a wide spectra. Therefore, an appropriate window function should be used in order to suppress the spreading of the frequency spectra. In the present study, we adopt the four-term Blackman-Harris window function (hereinafter $4 \mathrm{BH}$ ) (Harris 1978). The characteristics of the $4 \mathrm{BH}$ are shown in Figure 5. The amplitude of the window function using 512 data samples is symmetric and has a finite value at both edges (Figure 5a). The spectrum obtained by the discrete Fourier transform (DFT) shows that the main lobe is wider in the frequency domain, whereas the levels of the side lobes are well suppressed near the main lobe (Figure 5b). The small leakage of the spectral power in the side lobes prevents the loss resulting from the passband filtering.

Figure 6 shows the results obtained by applying the $4 \mathrm{BH}$ window to each FFT frame in the time domain. 


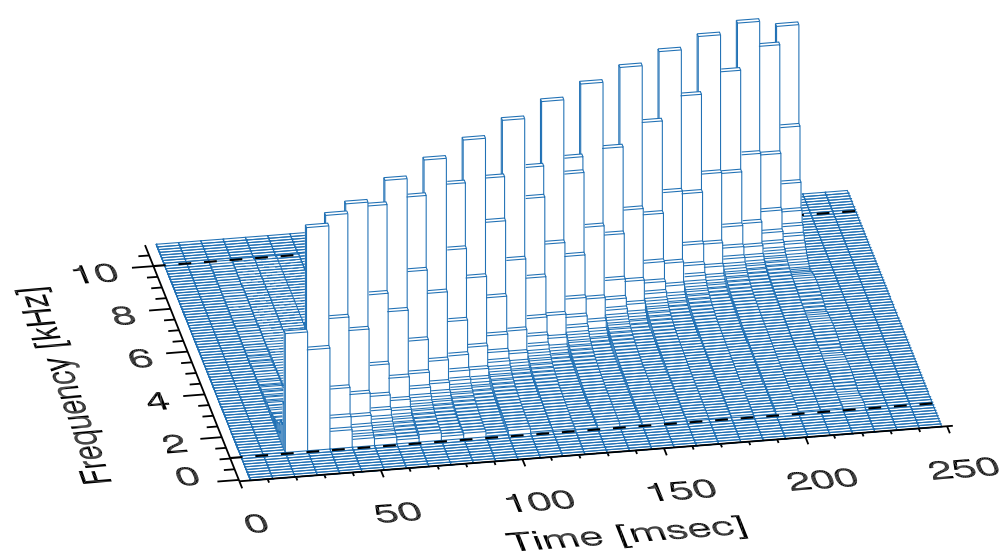

Figure 3 Time-frequency spectrograms for the model chorus wave. The dashed lines are the upper and lower cutoff frequencies of the passband filter.

The phase errors that exist over a frame in Figure $4 \mathrm{~b}$ are removed by taking the window function (Figure $6 \mathrm{~b}$ ). However, the amplitudes of the side lobes do not become completely zero in the frequency domain. Spectral leakage still remains at the tail of the spectra. This leakage is filtered through passband filtering, resulting in phase errors at the edges of the frame.

In order to remove the phase errors, we adopt overlapping processing of the FFT frame. For simplicity of waveform processing, we use $50 \%$ overlapping of the frame. In order to perform the overlapping processing, we multiply one FFT frame in the entire waveform by the $4 \mathrm{BH}$ window. We then obtain the waveform in the time domain by IFFT after applying passband filtering. Since the amplitudes of the waveform influence the multiplied window function in the time domain, a processing that multiplies the waveform by an inverse function of the window function is required. Due to the passband filtering, a certain discontinuity remains at both edges of the waveform. We then remove one-quarter of the data length at both edges of the

(a)

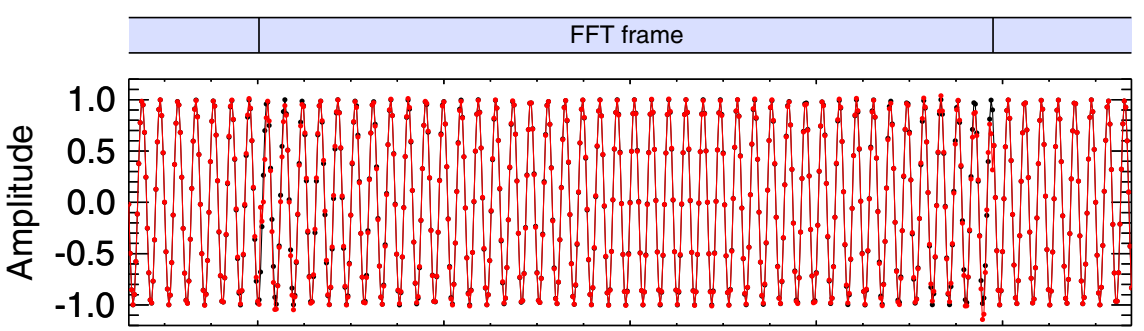

(b)

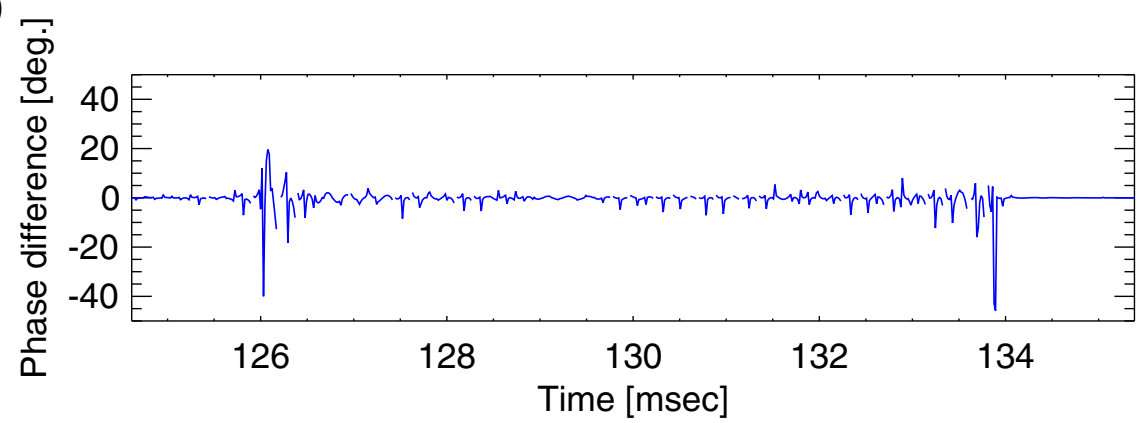

Figure 4 Wave amplitudes and phase differences. (a) Amplitudes of the model chorus waves. Waveform to which passband filtering is applied (red). The original waveform is plotted for comparison (black). The dots indicate the sampling data points. The FFT frames are shown at the top. (b) The instantaneous phase differences of the waveforms in (a) 
(a)

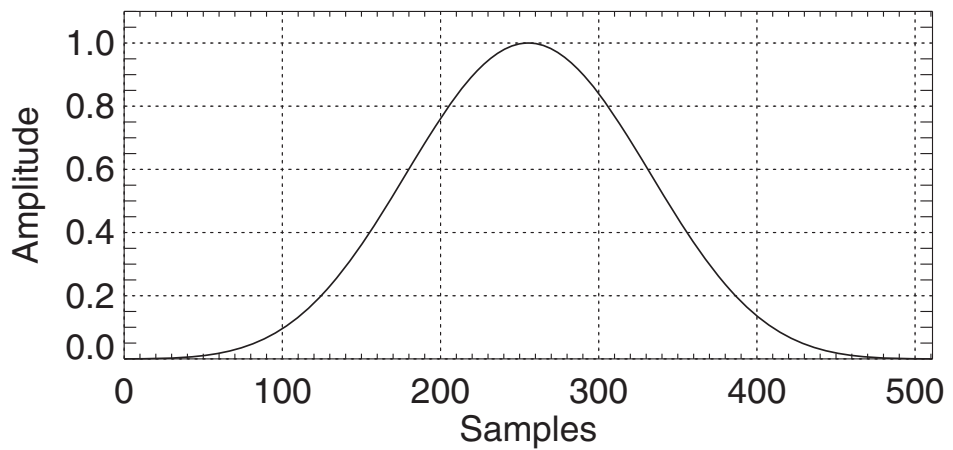

(b)

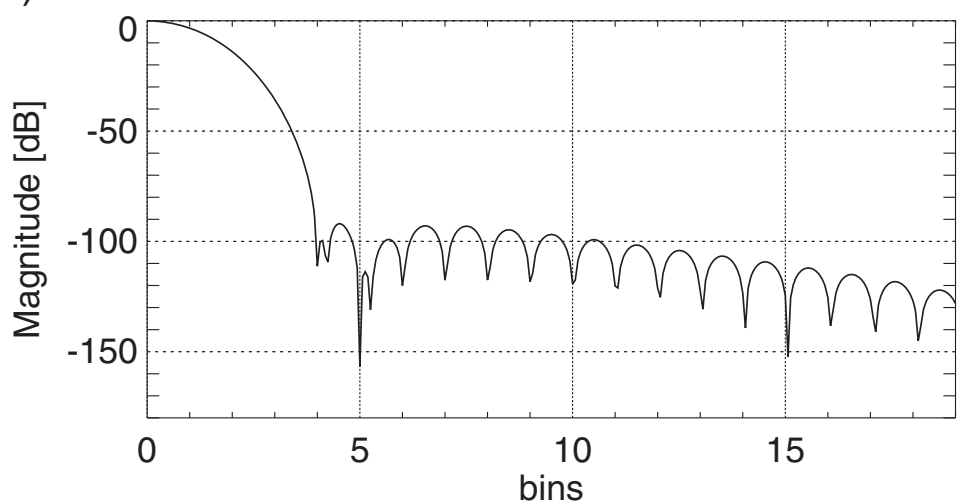

Figure 5 Characteristics of the four-term Blackman-Harris window function. (a) Amplitudes for 512 samples. (b) A portion of frequency responses (positive frequencies) by DFT.

frame where significant discontinuities are present and use the center half-length of the obtained waveform. The overlapped sequence is shown schematically at the top of Figure $7 \mathrm{a}$. The hatched lengths are removed through the processing. Thereafter, the $50 \%$ overlapped frame is used as subsequent FFT processing. The waveform obtained through the sequential overlapped processing is shown in Figure 7. Unlike the case of no overlapping shown in Figure 4, no phase errors exist in the time interval. Note that, even using the method with the $4 \mathrm{BH}$ window and the overlapping processing, phase errors with a few degrees occur locally. The appearance frequency of the phase error and its magnitude are remarkable at the frequency band of the chorus waves, which is close to the upper or lower frequency cutoff. The $50 \%$ overlapping processing of FFT approximately doubles the computation time compared to that without overlapping.

The waveform processing is summarized as follows:

1. Using 512 data points $(7.8 \mathrm{~ms})$ in one FFT frame

2. Multiplying the frame by the four-term Blackman-Harris window

3. Conversion to the frequency domain by FFT

4. Calibration using transfer functions
5. Using a passband filter $\left(f=0.1 \sim 1.0 f_{\text {ce }}\right)$ to obtain the frequency band of the chorus wave

6. Conversion to the time domain by IFFT

7. Using the waveform after removing one-quarter length from both edges of the frame

\section{Pseudo-measurement of WPIA in simulation}

We evaluate the effectiveness of waveform processing in the WPIA measurement by using chorus waves reproduced in simulation. The WPIA measurement in the simulation is described in Katoh et al. (2013), and we conduct the WPIA measurement in the same processing. The full-particle simulation code is used for the generation of whistler-mode chorus waves (Hikishima et al. 2009a, 2009b, 2010). Maxwell's equations and equations of relativistic particle motion are self-consistently solved in the simulation. The propagation of whistler-mode waves is assumed in the one-dimensional model along the ambient dipole magnetic field line near the geomagnetic equator. Two species of particles are used in the system: cold electrons form an isotropic Maxwellian distribution, and energetic electrons form an anisotropic bi-Maxwellian distribution $\left(V_{\mathrm{th} \perp}^{2} / V_{\mathrm{th} \|}^{2}-1=1.3\right)$ with a loss cone, where $V_{\text {th } \perp}$ and $V_{\text {th } \|}$ are perpendicular and parallel components, 
(a)

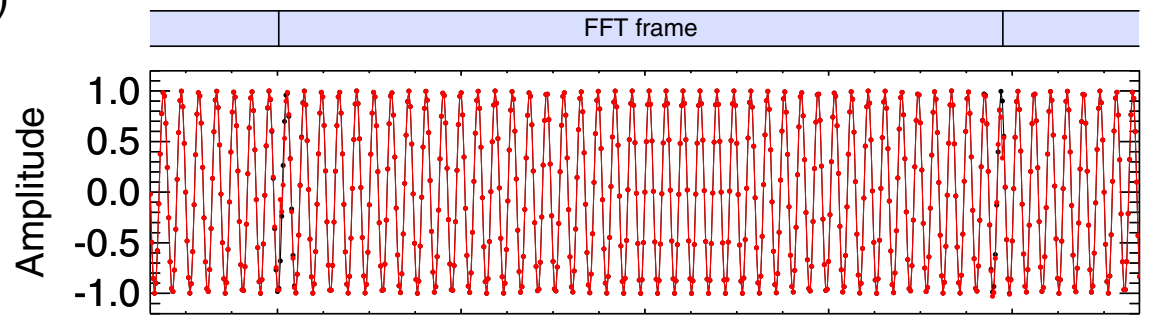

(b)

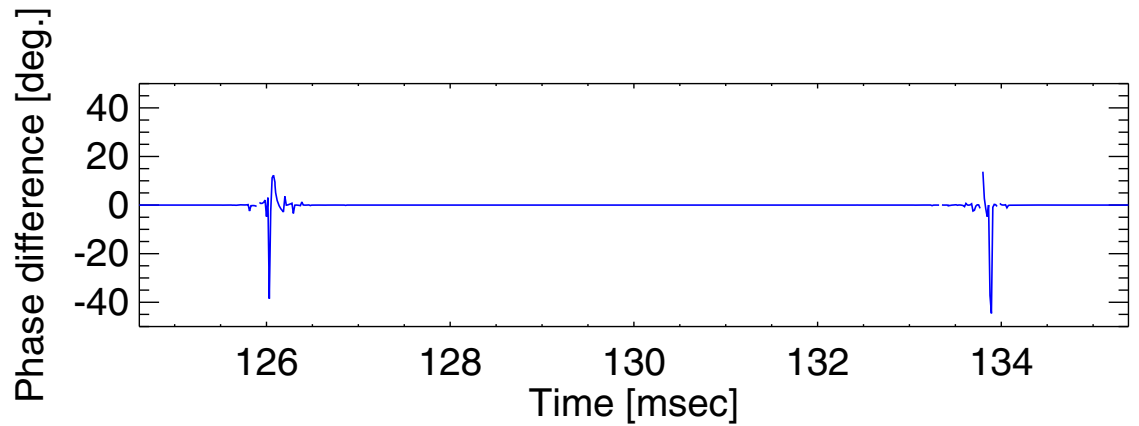

Figure 6 Wave amplitudes and phase differences. Analogous to Figure 4: (a) Amplitudes of the model chorus wave. Waveform to which passband filtering is applied after taking the window function (red). The original waveform is plotted for comparison (black). The dots indicate the sampling data points. The FFT frames are shown at the top. (b) Instantaneous phase differences of the waveforms in (a).

(a)
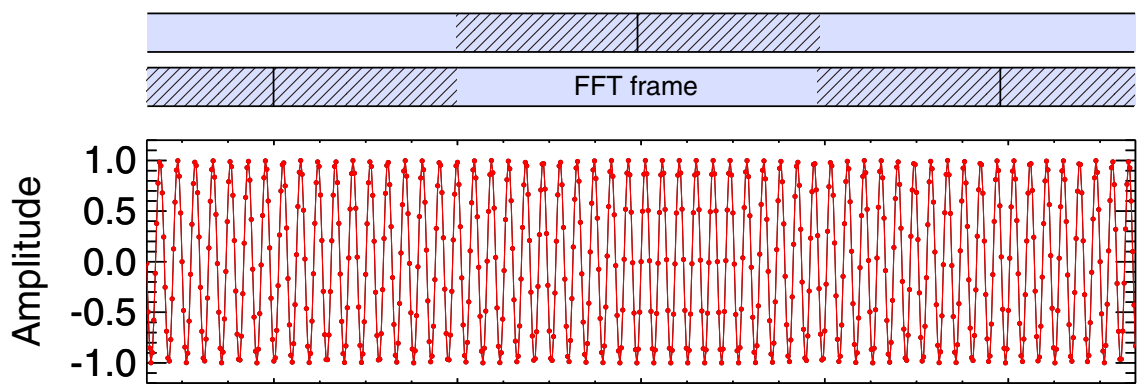

(b)

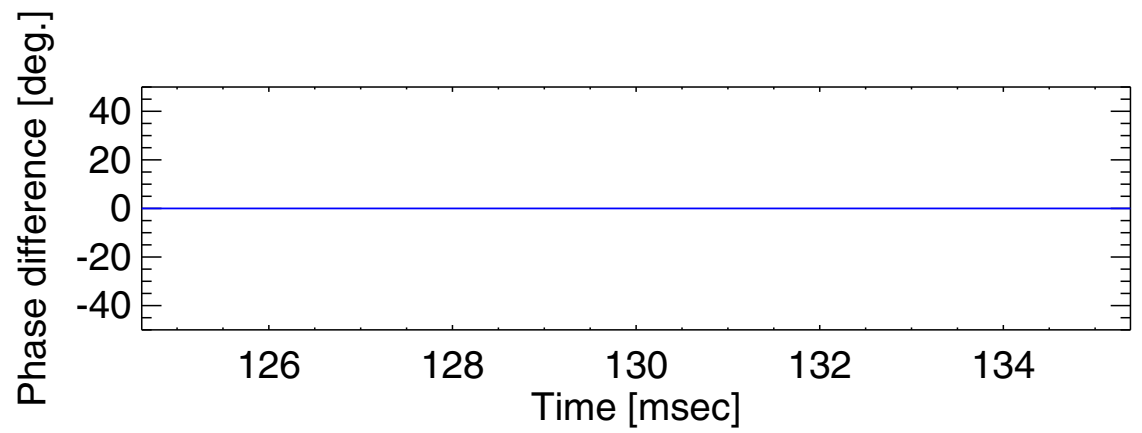

Figure 7 Wave amplitudes and phase differences. Analogous to Figure 4: (a) Wave amplitudes of model chorus waves. Waveform to which passband filtering is applied after taking the window function and is overlapped in the time domain (red). The original waveform is plotted for comparison (black). The dots show sampling data points. The FFT frames are shown at the top. (b) Instantaneous phase differences of the waveforms in (a). 
Table 2 Simulation parameters

\begin{tabular}{lc}
\hline Parameter & Value \\
\hline Time step & $0.005 \Omega_{\mathrm{e} 0}^{-1}$ \\
Grid spacing & $0.01 \mathrm{c} \Omega_{\mathrm{e} 0}^{-1}$ \\
Number of grids & 32,768 \\
Total number of energetic electrons & $536,870,912$ \\
Total number of cold electrons & $134,217,728$ \\
Density ratio of energetic electrons to cold electrons & $7.40 \times 10^{-3}$ \\
Thermal velocities of energetic electrons & $0.24 c, 0.36 \mathrm{c}$ \\
at the equator, $V_{\text {th } \|}, V_{\text {th } \perp}$ &
\end{tabular}

respectively, of thermal velocities. The density ratio of energetic to cold electrons is $7.4 \times 10^{-3}$. Background ions are assumed to satisfy the charge neutralization. The other simulation parameters are listed in Table 2.

Figure 8 shows the simulation results, which we use in the pseudo-WPIA measurement. In the simulation, whistler-mode chorus waves are generated through nonlinear cyclotron resonant interaction between background thermal noise and anisotropic energetic resonant electrons. The wave growth starts from thermal fluctuations through a linear wave growth by temperature anisotropy of energetic electrons. Then, the generated whistler-mode waves propagate toward higher latitudes from near the magnetic equator (Figure 8a). Figure $8 \mathrm{~b}$ shows the time-frequency spectrogram of generated rising-tone chorus waves at position $h=40 c \Omega_{\mathrm{e} 0}^{-1}$, where $c$ is the speed of light and $\Omega_{\mathrm{e} 0}$ is the equatorial electron gyrofrequency. Coherent rising-tone waves are formed due to the nonlinear wave growth, once the growing wave amplitude exceeds a threshold (Omura et al. 2009).

We then conduct the pseudo-WPIA measurement using the rising chorus element obtained in the simulation. The passband filtering and overlapping method are applied in the WPIA measurement. In order to conduct the WPIA measurement, we calculate the quantity of the energy transfer $W$ using the instantaneous waveform at a fixed point, $h=40 c \Omega_{\mathrm{e} 0}^{-1}$, and the velocity vectors of resonant electrons passing through the point. We focus on the component of $W$ that is perpendicular to the ambient magnetic field. The $W$ component is given by the formula $W=e \boldsymbol{E}_{\mathrm{W}} \cdot \boldsymbol{v}_{\perp}$ for the transverse component, where $e$ is the electron charge, and $\boldsymbol{E}_{\mathrm{w}}$ and $\boldsymbol{v}_{\perp}$ are perpendicular components of the wave electric field and the velocity vector of the electron, respectively. In order to statistically evaluate the quantity of energy transfer, we then calculate the integrated $W_{\text {int }}$ during a certain time interval. In the simulation, the intense generated rising chorus waves propagate toward the positive $h$ region and resonate with counter-streaming energetic electrons having negative parallel resonance velocity with respect to the

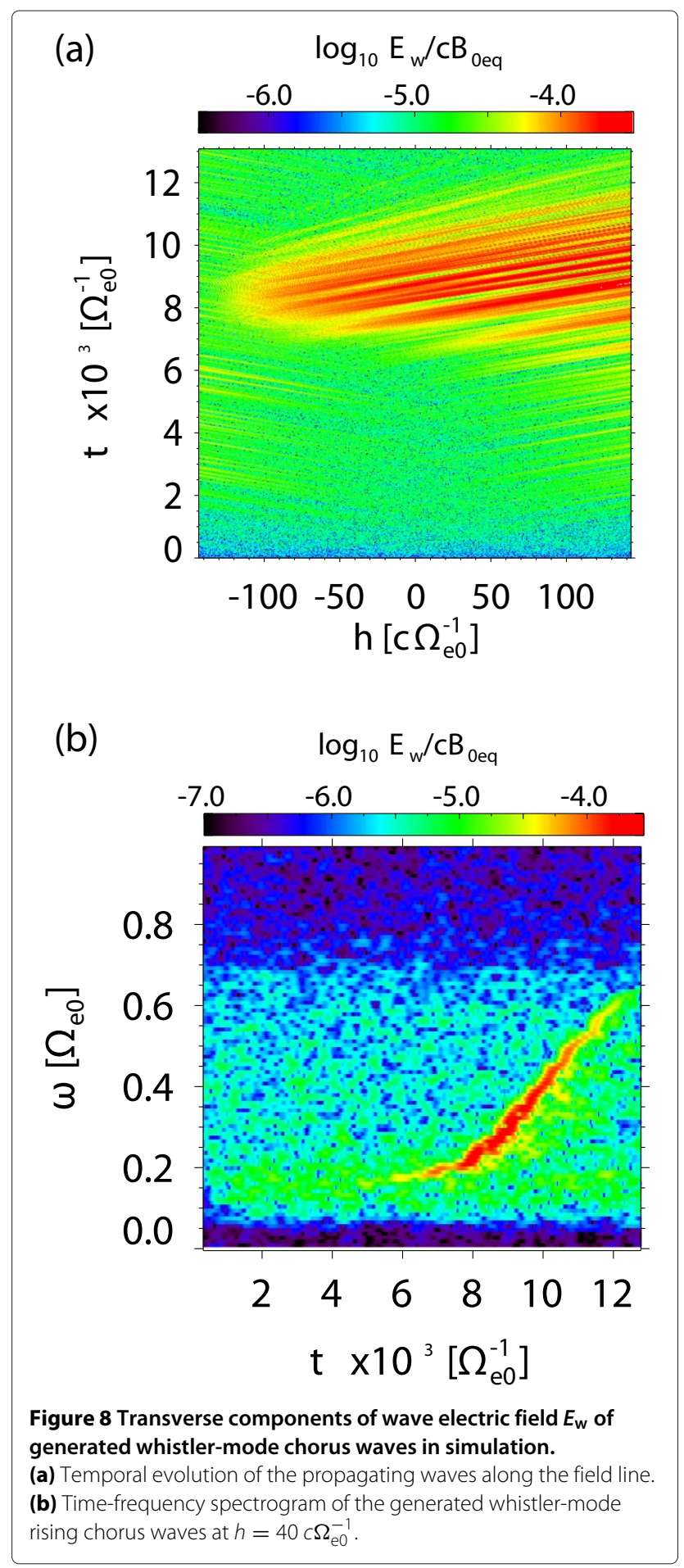

ambient magnetic field direction. Although only electrons near a specific resonance velocity involve cyclotron resonance with whistler-mode waves, all electrons are used in the calculation of $W_{\text {int }}$.

Figure 9 shows the results of the pseudo-WPIA measurement in the simulation. The integrated $W_{\text {int }}$ is plotted 


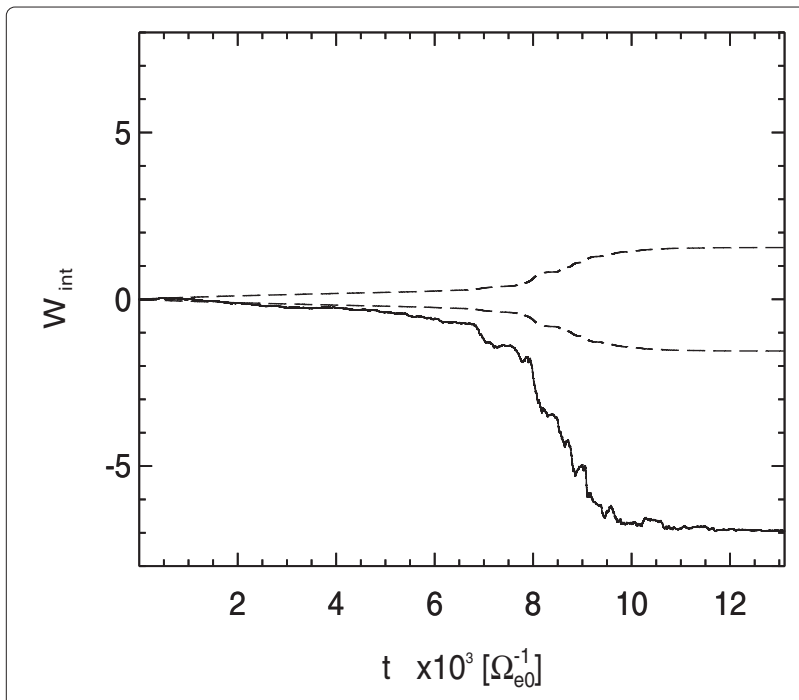

Figure 9 Time evolution of $W_{\text {int }}$ calculated using waveform of reproduced chorus waves and energetic electrons in simulation. The dashed lines indicate the standard deviation $2 \sigma_{\mathrm{w}}$.

as a function of time. In order to evaluate the statistical significance of $W_{\mathrm{int}}$, the standard deviations $2 \sigma_{\mathrm{w}}$ given in Equation 2 are plotted (dashed line). Once coherent rising chorus waves are generated $\left(t>6,000 \Omega_{\mathrm{e} 0}^{-1}\right)$, the absolute value of $W_{\text {int }}$ increases significantly above $2 \sigma_{\mathrm{w}}$. The transition of $W_{\text {int }}$ toward negative values implies that an energy transfer occurs from energetic electrons to chorus waves.

In order to examine the waveform processing, the amplitudes of dummy MSW and ECH waves are added. The pseudo-waveforms of the MSW and ECH waves are superposed on chorus waveform reproduced in the simulation. We assume the superposed waves in the frequencies of $0.6 f_{\mathrm{LHR}}$ for MSW and $1.5 f_{\text {ce }}$ for $\mathrm{ECH}$ waves. The wave amplitudes are comparable to that of chorus waves and are added to one component of transverse wave electric fields in the simulation. We note that the MSW and $\mathrm{ECH}$ waves do not resonate with electrons in the onedimensional simulation, namely no phase correlation with electrons. The added waves only give an amplitude modulation to chorus waves. We then apply the passband filtering and overlapping method for the WPIA measurement in the simulation. Figure 10 shows the $W_{\text {int }}$ with passband filtering (blue) and with passband filtering and overlapping method with the $4 \mathrm{BH}$ window function (red). The dashed lines indicate the standard deviations $2 \sigma_{\mathrm{w}}$. After chorus waves appear, the quantity of both $W_{\text {int }}$ is similar because the chorus wave amplitude is larger compared to fluctuations due to the phase errors. Before chorus waves appear, in case using only the passband filtering, the quantity of $W_{\text {int }}$ is close to the $2 \sigma_{\mathrm{w}}$. In case using the processing of the passband filtering and the overlapping, the absolute value of $W_{\text {int }}$ is larger than the $2 \sigma_{\mathrm{w}}$, as a result

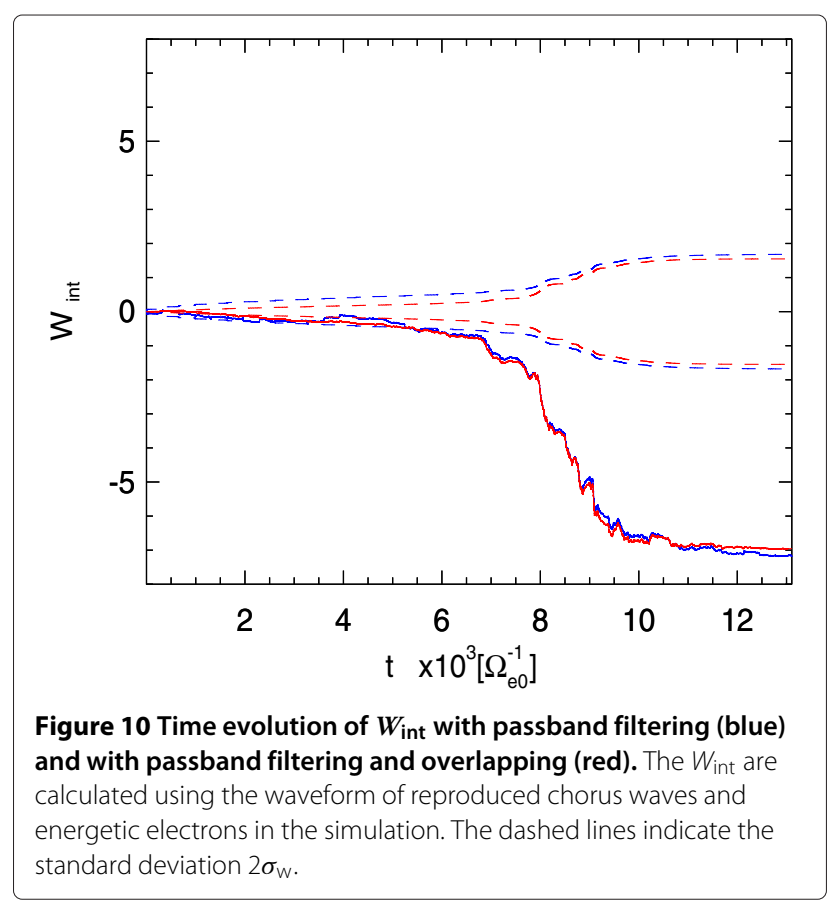

that the absolute value of the standard deviation becomes small. By applying the implementation of the overlapping method, the unnecessary discontinuity of the wave phase is removed. The processing method makes the larger statistical significance, giving a more accurate evaluation of the energy transfer.

\section{Conclusions}

The WPIA is a software function installed on the ERG satellite. In the WPIA calculation, we presented a processing method to reduce the errors of the wave phase in order to accurately evaluate the statistical significance of the energy transfer. The WPIA processing using the FFT and IFFT is costly. Moreover, the proposed overlapping processing of the waveform requires more computation time compared to that without overlapping. The computation resources (e.g., CPU clock, memory size, and bus width) are limited for the onboard data processing. We next address the evaluation of data processing speed and optimization of the software algorithm of the WPIA.

The target of the WPIA measurement is to evaluate the energy transfer between chorus waves and the resonant electrons. The ECH and MSW may affect particle motions. The particle fluctuations give uncertainty evaluation of $W_{\text {int. }}$. From spectral information, we therefore avoid the WPIA calculation during a period that the $\mathrm{ECH}$ and MSW exist. In addition, considering the resonance condition between waves and particles, we can expect that the particle energies influenced by ECH and MSW are almost different from those interacting with chorus waves. 
We select the kinetic energy of electrons for the WPIA calculation by referring the cyclotron resonance condition with chorus waves. We are planning to use other information such as the plasma density for the evaluation of the resonance condition, by referring to the linear dispersion of the whistler wave mode. The influence of ECH and MSW waves on the WPIA measurement should be evaluated by a self-consistent multi-dimensional simulation and is left for the future study.

In the particle simulation, a coherent rising-tone element with the enhanced nonlinear wave growth $(t>$ $\left.6,000 \Omega_{\mathrm{e} 0}^{-1}\right)$ is formed after moderate linear wave growth of incoherent whistler-mode waves. The WPIA measurement demonstrated significant energy transfer from an energetic electron to a whistler-mode chorus wave which occurs during the generation of the coherent rising chorus element. During the period of $t<6,000 \Omega_{\mathrm{e} 0}^{-1}$ in which the linear wave growth occurs near the frequency $\omega=0.2 \Omega_{\mathrm{e} 0}$, the WPIA measurements indicate the gradual increase of the quantity $W$, exceeding the $2 \sigma_{\mathrm{w}}$ (Figure 10 ).

The main goal of the WPIA is to directly measure the quantity of energy transfer between whistler-mode chorus waves and resonating energetic electrons in the space plasma environment. In order to statistically evaluate the quantity of energy transfer, it is important to obtain accurate phase angles of the wave vector and the velocity vector of a particle. In addition to chorus waves, various other waves are included in the observed waveform, which affects the accuracy of the calculated quantity of energy transfer. The wave modes are removed through the passband filtering. However, the removed spectrum causes undesirable phase angle errors of the waveform. In order to reduce the phase errors, the overlapping processing is applied using an appropriate window function. The processing shows that the phase angle errors are improved successfully. The proposed waveform processing method is necessary in order to reduce the phase error and is applicable to the calculation of theWPIA.

Competing interests

The authors declare that they have no competing interests.

\section{Authors' contributions}

$\mathrm{MH}$ carried out the analyses of the work. YK and HK were involved in the discussion of the results and revised the manuscript. All authors read and approved the final manuscript.

\section{Acknowledgements}

This paper is dedicated to Prof. Takayuki Ono, who passed away on 21 December 2013. The computations in the present study were performed on the KDK system of RISH and ACCMS at Kyoto University. This work was supported by grant-in-aid 23224011 of the Ministry of Education, Culture, Sports, Science and Technology in Japan.

\section{Author details}

${ }^{1}$ Department of Geophysics, Graduate School of Science, Tohoku University, Miyagi 980-8578, Japan. ${ }^{2} 2$ Solar-Terrestrial Environment Laboratory, Nagoya University, Nagoya, Aichi 464-8601, Japan. ${ }^{3}$ Research Institute for Sustainable Humanosphere, Kyoto University, Kyoto 611-0011, Japan.
Received: 7 January 2014 Accepted: 17 June 2014

Published: 1 July 2014

\section{References}

Burtis WJ, Helliwell RA (1969) Banded chorus-a new type of VLF radiation observed in the magnetosphere by OGO 1 and OGO 3. J Geophys Res 74:3002

Fukuhara H, Kojima H, Ueda Y, Omura Y, Katoh Y, Yamakawa H (2009) A new. instrument for the study of wave-particle interactions in space: One-chip Wave-Particle Interaction Analyzer. Earth Planets Space 61:765

Gurnett DA (1976) Plasma wave interactions with energetic ions near the magnetic equator. J Geophys Res 81(16):2765-2770

Harris FJ (1978) On the use of windows for harmonic analysis with the discrete fourier transform. Proc IEEE 66:51-83

Hikishima M, Yagitani S, Omura Y, Nagano I (2009a) Full particle simulation of whistler-mode rising chorus emissions in the magnetosphere. J. Geophys. Res 114:A01203. 10.1029/2008JA013625

Hikishima M, Yagitani S, Omura Y, Nagano I (2009b) Coherent nonlinear scattering of energetic electrons in the process of whistler mode chorus generation. J Geophys Res 114:A10205. 10.1029/2009JA014371

Hikishima M, Omura Y, Summers D (2010) Microburst precipitation of energetic electrons associated with chorus wave generation. Geophys Res Lett 37:07103. 10.1029/2010GL042678

Katoh Y, Kitahara M, Kojima H, Omura Y, Kasahara S, Hirahara M, Miyoshi Y, Seki K, Asamura K, Takashima T, Ono T (2013) Significance of Wave-Particle Interaction Analyzer for direct measurements of nonlinear wave-particle interactions. Ann Geophys 31:503-512. 10.5194/angeo-31-503-2013

Kennel CF, Scarf FL, Fredricks RW, McGehee JH, Coroniti FV (1970) VLF electric field observations in the magnetosphere. J Geophys Res 75:6136-6152

Meredith NP, Horne RB, Iles RH, Thorne RM, Heynderickx D, Anderson RR (2002) Outer zone relativistic electron acceleration associated with substorm-enhanced whistler mode chorus. J Geophys Res 107(A7):1144. 10.1029/2001JA900146

Meredith NP, Horne RB, Thorne RM, Anderson RR (2009) Survey of upper band chorus and ECH waves: implications for the diffuse aurora. J Geophys Res 114(A07218). 10.1029/2009JA014230

Miyoshi Y, Ono T, Takashima T, Asamura K, Hirahara M, Kasaba Y, Matsuoka A, Kojima H, Shiokawa K, Seki K, Fujimoto M, Nagatsuma T, Cheng CZ, Kazama Y, Kasahara S, Mitani T, Matsumoto H, Higashio N, Kumamoto A, Yagitani S, Kasahara Y, Ishisaka K, Blomberg L, Fujimoto A, Katoh Y, Ebihara Y, Omura Y, Nose M, Hori T, Miyashita Y, et al. (2013) The energization and radiation in geospace (ERG) project. In: Summers D, Mann IR, Baker DN, Schulz M (eds) Dynamics of the earth's radiation belts and inner magnetosphere. American Geophysical Union, Washington, D.C

Omura Y, Hikishima M, Katoh Y, Summers D, Yagitani S (2009) Nonlinear mechanisms of lower-band and upper-band VLF chorus emissions in the magnetosphere. J Geophys Res 114(A07217). 10.1029/2009JA014206

Santolík O, Pickett J, Gurnett D, Maksimovic M, Cornilleau-Wehrlin N (2002) Spatiotemporal variability and propagation of equatorial noise observed by cluster. J Geophys Res 107(A12):1495. 10.1029/2001JA009159

Summers D, Thorne RM, Xiao F (1998) Relativistic theory of wave-particle resonant diffusion with application to electron acceleration in the magnetosphere. J Geophys Res 103(A9):20487-20500

Tsurutani BT, Smith EJ (1974) Postmidnight chorus: a substorm phenomenon. J Geophys Res 79(1):118-127

doi:10.1186/1880-5981-66-63

Cite this article as: Hikishima et al.: Evaluation of waveform data processing in Wave-Particle Interaction Analyzer. Earth, Planets and Space 2014 66:63. 